complicated systems of mountain winds with a diurnal component are then described and analysed theoretically. Lastly, he examines effects on the wind ficld which are mechanically produced by mountains or escarpments, for example, the generation of the föhn, the bora, the mistral and lee-wave systems.

In the final chapter on climatic fluctuations, H. $H$. Lamb, after a short sumunary of the evidence of changes, ice ages and warm epochs, in the remote past, doals in more detail with the main climatic phases of post-glacial time, the past 10,000 years, concluding this section on elimatic history with an analysis of observations in tho era of meteorological instruments. An account is then given of circulation studies, the preparation of maps of prevailing wind and pressure patterns in different years and epochs, which may reveal the mechanism of climatic changes. The results of the analysis of the author's own series of maps and indices for the period from 1750 and his current work on the extension of the circulation analysis to years before 1750 are described. The chapter ends with a short summary of the causes and course of climatic variation.

This is a very good book which will be valuable to the meteorologist as woll as to the climatologist.

James Paton

\section{BOUNDARY LAYERS FOR ENGINEERS}

\section{Boundary Layers of Flow and Temperature}

By A. Walz. Edited and translated from the German by Hans J. Oser. Pp. xv +297. (MIT Press: Cambridge, Massachusetts, and London, January 1970.) 1648.

BOUNDARY-LAYER theory has formed a major part of fluid dynamics since Prandtl's famous paper was published in 1904. Its first achievement was to resolve the conflict between the hydromechanics of the mathematicians and the hydraulics of the engineers; the formor subject was elegant, extensive and founded on a plausible idealization of the behaviour of fluids; the latter was crude, empirical and fragmentary, but much more reliable as a predictor of the actual behaviour of real fluids. Prandtl showed that the small viscosity of these fluids, though negligible in its direct effect over a large part of the domain of flow, could have substantial indirect offects, emanating from the thin layer of fluid adjacent to the solid boundaries of the domain. The subsequent detailed investigations of particular boundary-layer flows completely clarified the qualitative phenomena which arise.

Boundary-layer theory has influenced engineering practico more through the understanding which it promotes than by providing quantitative methods of design; for the differential equations were too difficult to solve exactly until computer mothods were devised; and the laws of turbulent transfer, which dominate most practical flows, are only now being discovered. In the past few years, however, fast computational procedures of the finite-difference variety have become available; and satisfactory mathematical models of turbulence have been developed for use with them. Boundary-layer theory is at last ready for usc by engineers.

The demonstration of the usability of boundary layer theory is one of the motives of Professor Walz's book. The material and methods of the book spring, however, from the pre-computer years during and after the Second World War. Thus, from the mathematical point of view, its proceduros are approximate rather than rigorous; and the properties of turbulence are incorporated by way of empirically based correlations of the behaviour of complete boundary layers. Though the use of the computer is now advocated for the execution of the calculation methods, the full potentialities of tho computer are not exploited.
Although the book is therefore likely to have come rather too late to influence engineering practice widely, its high quality as a piece of exposition deserves recognition. The treatmont is scholarly and, granted the basic limitation just mentioned, it is both comprehensive in coverage and fair in evaluation. The author has taken much trouble to cnsure that his methods of computation can be understood and practically applied. Engineers will find his methods to be fast, fairly easy to understand, and, within their rather narrow limits of validity, sufficiontly accurate.

A few critical remarks should be made, however. Tho translation from the German is excellent in general, but marred by some inelegancies of construction or diction; thus the choice of the word "clot" for a coherent body of fluid in turbulent motion has led to some comic sentences. More serious is the disregard of tho possibility that the solutions of the integral equations, which aro Walz's preferred medium of computation, may not be unique; yet this often occurs when the velocity profile is "wcighted", in the formation of theso equations, by some function of the velocity, $u$; and it prevents the computation process from being completed. Because "weighting", functions of the cross-stream distance $y$ are frce from this disability, the author's preference for $u$-functions over $y$-functions may mislead his readers; it can be explained only by the fact that the author has not yet tried to apply his method to problems in which a maximum is present in the $u(y)$ profile, giving rise to two values of $y$ for one of $u$. Again, Walz recommends von Karman's modifications of the Prandtl-Taylor relation between friction and heat transfer, without mentioning that it brings very little improvement, and is inferior to the more recent formulae of Deissler and others.

In spite of these blemishes, tho book remains a valuable contribution to the boundary-layer literature. It was well worth translating.

D. B. Spalding

\section{PARTICLE TRANSFER}

Introduction to the General Theory of Particle Transfer By V. Kourganoff. (Documents on Modern Physies.) Pp. xiii +216. (Gordon and Breach: New York and London, January 1970.) $\$ 16.80 ; 140 \mathrm{~s}$.

THIs book is an attempt to present a graduate student at the beginning of his course with a simple account of the way in which problems in particle transfer can be formulated in terms of differential equations. It provides a meticulous account of the way in which a field of moving particles interacts with a material medium. The mathematical analysis has deliberately been kept simple so as to focus attention on the physical concepts and to allow those who intend to specialize in, say, transport of neutrons to see something of the relationship of their chosen field with that of radiative transfer.

A book of this kind has been needed for some time, but it is not clear that this one properly fills the gap. The level of knowledge assumed is often extremely low-it should hardly be necessary to have to give a proof of Gauss's theorem to graduates-and a good deal of space is occupied by recording minor steps in the argument which the reader might be expected to supply for himself. It is also disappointing that the physics of the microscopic interactions of neutrons and photons with matter is not treated more comprehensively. The mathematical shape of the source and sink terms which determine the complexity of transfer theory depends on these interactions. This book will only give the student a very limited understanding of the relationship of tho physies to the more mathematical problems treated in other texts. It is, morcover, extremely expensive for its size, the price being one which few students are likely to want to pay.

I. P. GRaNT 